\title{
Hemorrhaging laparoscopic partial nephrectomy — feasibility of a novel simulation model
}

Avril J. Lusty, $\mathrm{MD}^{1}$; Joanne Bleackley, $\mathrm{MD}^{2}$; Matthew Roberts, $\mathrm{MD}^{1}$; James Watterson, $\mathrm{MD}^{1}$; Isabelle Raîche, $\mathrm{MD}^{3}$

${ }^{1}$ Department of Urology, The Ottawa Hospital, Ottawa, ON, Canada; ${ }^{2}$ Department of Anaesthesia, The Ottawa Hospital, Ottawa, ON, Canada; ${ }^{3}$ Department of Surgery, The Ottawa Hospital, Ottawa, ON, Canada

Cite as: Lusty AJ, Bleackley J, Roberts M, et al. Hemorrhaging laparoscopic partial nephrectomy - feasibility of a novel simulation model. Can Urol Assoc J 2021 November 18; Epub ahead of print. http://dx.doi.org/10.5489/cuaj.7324

Published online November 18, 2021

Corresponding author: Dr. Avril J. Lusty, Department of Urology, The Ottawa Hospital, Ottawa, ON, Canada; alusty@qmed.ca

***

\section{Abstract}

Introduction: Intraoperative surgical complications pose significant potential risks to patients. Uncontrolled bleeding during laparoscopic partial nephrectomy is one such event that requires collaboration and communication between surgical team members. We developed and evaluated a multidisciplinary surgical simulation scenario and model of intraoperative hemorrhage during a laparoscopic partial nephrectomy to facilitate the practice of these crucial non-technical skills. Methods: A simulation scenario using a novel, titratable bleeding partial nephrectomy model was developed. The operating room simulation consisted of an intubated mannequin placed in the lateral decubitus position and laparoscopic renal model. The multidisciplinary simulation scenario included anesthesia and urology residents and progressed from bleeding to a pulseless electrical activity arrest. The degree of renal model bleeding was modified based on the progression of the urology resident. After the scenario participants were debriefed and completed a post-simulation survey assessing: 1) their perception of the simulated scenario; and 2) their teaching of non-technical skills in their residency training.

Results: The porcine model was successfully reproduced for nine consecutive weeks and functioned well to simulate bleeding from a laparoscopic partial nephrectomy site; the bleeding was able to be titrated based on resident progression and excision of the simulated tumor. All residents stated the scenario was valuable to assess and improve non-technical surgical skills and that their exposure to practice non-technical skills in their existing curriculum could be improved. 
Conclusions: Simulating an intraoperative bleeding partial nephrectomy, combined with an intraoperative crisis scenario, is a feasible, immersive, and reproducible model and can challenge residents' non-technical skills.

\section{Introduction}

Surgeons, anesthesiologists, and other surgical team members must have both strong technical skills and non-technical skills such as situational awareness, leadership, communication and decision making ${ }^{1}$ in order to function safely and effectively in the operating room. Inadequate non-technical skills can lead to intraoperative complications with communication breakdown cited as one of the main causes of intraoperative errors. ${ }^{2}$ Fortunately, studies have shown that simulation and simulation-based team training (SBTT), involving multidisciplinary operative teams, have been successfully used to train both technical and non-technical skills in operative simulation scenarios. ${ }^{2,3}$

Most previously described laparoscopic urology scenarios have included simulated renal vein injuries and inferior vena cava injuries using porcine kidney, silicone gel or threedimensional printed technology. ${ }^{4-7}$ While some of these models have been used in SBTT scenarios ${ }^{6,8,9}$ to allow for practice and training of non-technical skills, many have been used solely for technical skill practice. ${ }^{4,5}$ Despite the availability of such described models, a recent Canadian survey of academic urology program stakeholders including program directors, academic staff, and residents, found programs have limited simulation experiences within their protected academic teaching time, even though most stakeholders agreed on the value of simulation to be utilized as a teaching method in urology. ${ }^{10}$

Based on the previously described simulation models and scenarios in urology ${ }^{6,9,11}$, we aimed to develop a renal model to challenge the non-technical skills of urology residents. A key feature of practicing non-technical skills in an operative crisis is to encourage residents, who may progress easily through a technical skill, to still challenge and practice their non-technical skills. Previous simulations have not included a bleeding model that can be titrated or made more challenging in real-time and be modified based on a learner's progress. ${ }^{4-7,9,10}$. We therefore aimed to include this adaptability into our SBTT of urology and anaesthesia residents in a hemorrhaging laparoscopic partial nephrectomy scenario.

The selection of a partial nephrectomy for the operative scenario creates a sense of urgency for the urology resident to limit warm ischemia time. Further, the addition of titratable blood flow through the renal model allows for visible worsening of hemorrhage and allows residents who are progressing with the partial nephrectomy technical skill to still be challenged with their non-technical skills while responding to increased intraoperative bleeding. This model was included in a multidisciplinary SBTT simulation to challenge the non-technical skills of 
urology residents with anaesthesia residents in a hemorrhaging, laparoscopic partial nephrectomy scenario that culminated in a pulseless electrical activity arrest.

The goal of this simulation was to pilot a novel, titratable, hemorrhaging, laparoscopic partial nephrectomy model to simulate an operative crisis. We also aimed to determine the exposure of urology residents to, as well as practice, their non-technical skills.

\section{Methods}

PGY 3-5 urology residents and PGY 1-5 anaesthesia residents participated in a hemorrhaging laparoscopic partial nephrectomy simulation scenario, including pre-briefing and debriefing of all residents. Of note, all PGY 3-5 urology residents were invited to voluntarily attend, and PGY 1-5 anaesthesia residents attended as part of their mandatory postgraduate medical education curriculum.

Each simulation took place at the University of Ottawa Skills and Simulation Centre (uOSSC). Both urology and anaesthesia residents were briefed that they would enter a simulated intraoperative scenario involving a laparoscopic partial nephrectomy and obtained handover from their counterparts in the operating room including relevant patient history, imaging and laboratory values. A surgical assistant, a circulating nurse and a scrub nurse were present as confederates during the simulation. Residents were not told which type of intraoperative events would take place during the scenario. The simulation, including pre-brief, scenario, and debrief were recorded. The initial intent of the simulations was to review the residents' non-technical skills with NOTSS framework ${ }^{1}$. The NOTSS framework is widely accepted to assess surgeons', as well as surgical teams', non-technical skills ${ }^{13}$. Further, this framework has been shown to successfully assess surgical trainees' non-technical skills in the competency-based structure of surgical residency training programs ${ }^{14}$. All urology residents performed a self-assessment with NOTSS scoring grid at the completion of the scenario and these would be compared to staff NOTSS assessments of the recorded videos. This NOTSS component of the project, comparing staff and resident self-assessments, was not completed as the numbers would be significantly underpowered for statistical analysis. Ethics approval was obtained through the University of Ottawa Quality Improvement board.

\section{Bleeding partial nephrectomy kidney model and operating room}

An ex-vivo porcine kidney, including renal artery, renal vein and renal pelvis and ureter, was used for each simulation. A defect was made at the upper pole of the kidney and a coloured Styrofoam ball was fixed in the renal parenchyma bed to simulate the renal tumour. A 14Fr Foley catheter was placed through punctured renal hilum and through the renal parenchyma towards the renal mass defect. To simulate bleeding from the renal tumour resection bed the Foley catheter was connected to irrigation tubing and a four-litre bag of water dyed red to simulate blood. The dyed irrigation fluid was placed under pressure and each kidney model was tested prior to the scenario for appropriate bleeding of the defect on the morning of each simulation. Any model that was deemed inadequate for the simulation was re-made and re- 
tested. Each scenario had a fresh ex-vivo porcine partial nephrectomy model. The models cost $\$ 9.07$ (CND), including $\$ 5.00$ for the porcine kidney, $\$ 3.57$ for irrigation tubing and $\sim \$ 0.50$ for the Styrofoam renal mass. A simulation technician from the uOSSC built and tested each model with the aid of one of the authors (AL). Two kidney models needed to be rebuilt after hemorrhagic testing was deemed non-satisfactory and were successfully re-done prior to the simulations. Each model took approximately one hour to build and test for modifiable hemorrhage. A total of ten kidney models were built, including seven for the scenario, two models that needed to be rebuilt for insufficient hemorrhage, and one that was made for the simulation dry run. The total cost of the simulations was $\sim \$ 82.56$ (CND).

The kidney model was attached inside the uOSSC laparoscopic abdominal box in an orthotopic fashion, with simulated small bowel placed medial to the renal hilum to simulate the duodenum and a bulldog clamp placed over the renal hilum to simulate vascular control (Figure 1). A high-fidelity Laerdal 3G SimMan mannequin was placed in the lateral decubitus position, with an abdominal roll placed behind the flank to simulate proper flank position. The laparoscopic abdominal box was placed in front of the mannequin, also in the lateral decubitus position, to simulate laparoscopic positioning.

\section{Scenario}

The scenario was jointly developed by urology and anesthesia faculty and fellows. The participating urology and anesthesia residents were briefed on the scenario and provided handover. Three anaesthesia residents and one urology resident were present for each simulation. One anaesthesia resident was the participant in the simulation, one anaesthesia resident played the role of the scrub nurse and the third gave handover to the participant anaesthesia resident. The urology resident was instructed to enter the operating room to assist with a laparoscopic partial nephrectomy. The urology resident was given handover by the urology fellow who acted as a surgical assistant, holding the laparoscopic camera, and providing guidance to the resident as needed. One simulation fellow acted as the circulating nurse and the other simulation fellow and anaesthesia staff were located in the control room.

The scenario was divided into four sections. The first portion ran from the time the resident entered the simulation to the beginning of the tumour excision (0-2 minutes). The vital signs at this time were stable with a normal blood pressure. As the resident continued to excise the tumour, minor bleeding started, triggered by unclamping the irrigation tubing to a half open position. Next, the vitals slowly dropped over the subsequent 2-6 minutes. This represented the second portion of the procedure simulating "minor bleeding". The third portion occurred over the subsequent 7-10 minutes, where the irrigation tubing was opened fully to allow for maximal flow of blood from the resection bed. This portion represented "maximal bleeding". The vital signs dropped to a peri-arrest event, where the patient became tachycardic and hypotensive. If the urology resident was performing the mass excision quickly, the scenario accommodated for this and the simulation was advanced accordingly through each phase with more significant hemorrhage. The final portion of the scenario was a pulseless electrical activity arrest, where the 
anaesthesia and urology resident had to recognize the event and that the patient had to be taken out of the lateral decubitus position to commence chest compressions. The scenario ended after at least one round of chest compressions were completed, where the surgical confederate announced that the urology team was able to open the abdomen and control the bleeding (Figure 2).

\section{Debriefing session}

Each simulation ended with a debriefing session and included one urology resident, three anaesthesia residents, an anaesthesia staff, a urology fellow and two simulation fellows. Each debriefing session was led by the urology simulation fellow and anaesthesia staff, both of whom are trained in the foundations of debriefing. The debriefing discussed themes of crisis resource management that were highlighted during the scenario, including communication, situational awareness, and resource allocation. The debriefing sessions followed a standardized PEARLS framework ${ }^{15}$, with each session highlighting different crisis resource management themes that were discussed by participants as described above. The urology residents then completed a survey, with open-ended questions, assessing the realism of the hemorrhaging laparoscopic partial nephrectomy model and the non-technical skills utilised, including communication and shared decision-making, during the scenario.

\section{Results}

The simulation took place on a weekly basis from January 2020- March 2020 and was scheduled to run until the middle of April with all PGY 3-5 urology residents participating. Simulation sessions were prematurely halted due to the Covid-19 pandemic. All four PGY 5 residents participated, as well as two PGY 3 and one PGY 4 residents, for a total of seven residents, each completing the simulation once, and $100 \%$ of residents completed the post-simulation survey. Residents stated they had previously participated in 0-5 laparoscopic operating room simulations and/or SBTT scenarios; three residents stated they participated in zero simulations, one resident participated in two simulations and three residents participated in five simulations. The reported goals, as stated by the residents, of these previously completed team-based sessions included to practice 1) technical laparoscopic skills and 2) problem solving. Only the PGY5 residents had experienced a single real-world clinical encounter of a hemorrhaging laparoscopic partial nephrectomy requiring open conversion.

The most useful themes of the simulation as reported by the residents were surgical decision-making during laparoscopic surgery, identifying communication barriers and practicing communication skills with anaesthesia (Table 1). When asked if the residents would be able to implement the skills they learned during their clinical practice, $100 \%$ of residents reported that the skills learned would be transferable to clinical practice and $86 \%$ stated these would be implemented by improved communication skills and awareness of future communication barriers (Table 1). 


\section{Discussion}

We performed a pilot study to determine the feasibility of a multidisciplinary, hemorrhaging, laparoscopic partial nephrectomy scenario and renal model that allowed residents to practice non-technical skills in a simulated operating room crisis. Our study found urology residents have had limited experiences to practice non-technical surgical skills in simulation and cited interdisciplinary communication as the most useful component of our simulation. The novel bleeding partial nephrectomy model was feasible to construct and functioned well with both reasonable costs and resources required to replicate the simulation for future scenarios.

Urology simulation curricula and bootcamps have been shown to increase trainee competence and decrease time to develop laparoscopic skills. As such, the evidence to support incorporating laparoscopic skills training into urology programs has long been acquired ${ }^{16-19}$ with the majority of Canadian urology programs offering laparoscopic simulation training. ${ }^{10}$ In realworld clinical practice however, laparoscopic surgery takes place in an operating room with multidisciplinary teams. The operating room consists of a dynamic multidisciplinary team environment where technical skills intersect with non-technical skills such as communication, leadership, situation awareness and shared decision-making. ${ }^{1}$ These non-technical skills have been cited as some of the main causes of intraoperative errors and complications. ${ }^{2,20}$ Similar to standard surgical skills, non-technical skills can be developed during simulation scenarios providing a safe, low-stakes environment to improve non-technical skills. Furthermore, training non-technical skills using SBTT scenarios has been shown to improve intraoperative behaviours and surgical decision-making. ${ }^{21}$ While SBTT remains a key mechanism by which to train and teach both technical skills and non-technical skills ${ }^{22-25}$, literature review has shown that few urology programs have incorporated the deliberate practice of non-technical skills, either within their programs or as multidisciplinary teams. ${ }^{10,26,27}$ A dynamic simulation scenario, like the one described, provides a fertile environment for urology residents to practice their non-technical surgical skills both at a junior and senior level of training. Surveys have highlighted that urology programs support simulation training, particularly for technical skill teaching of junior residents ${ }^{10}$ however non-technical skill training is beneficial and should be practiced at all levels of training including junior and senior resident training level.

The responses regarding simulation experiences from our cohort mirror that of recently published literature. ${ }^{10,26}$ The urology residents from this pilot study responded that they have had limited multidisciplinary simulation exposure, ranging from zero to five simulations. Of those simulations, the majority took place during their junior surgical training years in simulated emergency department scenarios or non-operating room scenarios. This further highlights the need to develop scenarios such as "the operating room crisis" where non-technical skills training can be increased. Interprofessional communication in the operating room between multidisciplinary team members is a vital non-technical skill with previous research identifying this as an area in need of improvement. ${ }^{2}$ Residents in our pilot study stated that practicing communication skills with anaesthesia was one of the most useful components of the simulation. 
This response speaks to the urgency the piloted simulation evoked and required increased and deliberate communication between urology and anaesthesia during the intraoperative crisis. This also demonstrates that the urology residents acknowledged the importance of good communication in a crisis in addition to the importance of technical skills and know-how.

Urologic laparoscopic simulations have used both porcine and simulated renal models to simulate bleeding renal veins, and inferior vena cava injuries but few of these have been used for SBTT. ${ }^{4,5,12,28}$ Of these studies, Lee et al and Abelshehid et al evaluated technical skills and nontechnical skills of both urology and anaesthesia residents in their SBTT scenarios of a renal vein injury and laparoscopic partial nephrectomy, respectively, both showing positive feedback but with non-modifiable models. ${ }^{6,9}$ To our knowledge, this is the first time a simulated renal model has been made for hemorrhaging to accommodate and vary according to the urology resident laparoscopic partial nephrectomy progression.

The cost of simulation models in previously published studies varies depending on materials used and operating costs that can range from $\$ 56$ (USD) to $\$ 264$ (USD). ${ }^{4,5,12}$ The hemorrhaging partial nephrectomy model for this study was able to be reproduced for each weekly scenario, with each model costing $\sim \$ 9.07$. The model was easily constructed, and reproducible. The model and scenario can be implemented in either a high-fidelity scenario using the SimMan mannequin or adaptable to a low-fidelity model. This simulation can be adapted to a low fidelity model by omitting the SimMan mannequin entirely and not requiring the anaesthesia equipment or monitors. The uOSSC is a state-of-the-art and well-equipped centre, however the equipment and supplies required to produce this model were deliberately chosen and are readily available for programs with limited resources or funding.

Our study is limited by the small number of residents that were able to participate. The goal was to have all senior urology residents participate in the higher-level simulation which would have been a total of twelve urology residents at the University of Ottawa. Future simulations could include informal assessment of residents' non-technical surgical skills after their debrief and further reinforce the non-technical skill domains practiced in the simulation, as well as expand to other urologic intra-operative crises such as $\mathrm{CO} 2 \mathrm{embolism}$ and crises in the prone position. SBTT scenarios, like the one described in this paper, can be incorporated in the future into educational curricula, both for core discipline training and for transition to discipline.

\section{Conclusions}

The novel hemorrhaging partial nephrectomy model was feasible to construct, low cost, reproducible, and provided varying degrees of hemorrhage. The simulation also highlighted the importance of practicing non-technical skills and crisis resource management skills in operating room simulations between a multidisciplinary team. The next steps for this simulation would be to validate this research, including the need to obtain face, content, and construct validity prior to its incorporation into educational programs. 


\section{References}

1. Flin R, O'Connor P and Crichton M: Safety at the Sharp End: A Guide to Non-Technical Skills. Aldershot: Ashgate; 2008.

2. Greenberg CC, Regenbogen SE, Studdert DM, et al: Patterns of Communication Breakdowns Resulting in Injury to Surgical Patients. J Am Coll Surg 2007; 204: 533-540.

3. Huffman EM, Anton NE, Athanasiadis DI, et al: Multidisciplinary simulation-based trauma team training with an emphasis on crisis resource management improves residents' non-technical skills. Surg (United States) 2021: 1-4. Available at: https://doi.org/10.1016/j.surg.2021.03.015.

4. Higuchi M, Abe T, Hotta K, et al: Development and validation of a porcine organ model for training in essential laparoscopic surgical skills. Int J Urol 2020: 1-10.

5. Monda SM, Weese JR, Anderson BG, et al: Development and Validity of a Silicone Renal Tumor Model for Robotic Partial Nephrectomy Training. Urology 2018; 114: 114120. Available at: https://doi.org/10.1016/j.urology.2018.01.030.

6. Lee JY, Mucksavage P and McDougall EM: Simulating laparoscopic renal hilar vessel injuries: Preliminary evaluation of a novel surgical training model for residents. $J$ Endourol 2012; 26: 393-397.

7. Smith B and Dasgupta P: 3D printing technology and its role in urological training. World J Urol 2019. Available at: https://doi.org/10.1007/s00345-019-02995-1.

8. Goldenberg MG, Fok KH, Ordon M, et al: Simulation-Based Laparoscopic Surgery Crisis Resource Management Training_-Predicting Technical and Nontechnical Skills. J Surg Educ 2018; 75: 1113-1119. Available at: http://dx.doi.org/10.1016/j.jsurg.2017.11.011.

9. Abdelshehid CS, Quach S, Nelson C, et al: High-fidelity simulation-based team training in urology: Evaluation of technical and nontechnical skills of urology residents during laparoscopic partial nephrectomy. J Surg Educ 2013; 70: 588-595. Available at: http://dx.doi.org/10.1016/j.jsurg.2013.04.009.

10. Lobo AM, Doucette S and Powers AGL: Stakeholder perspectives and status of surgical simulation and skills training in urology residency programs in Canada. Can Urol Assoc J $2019 ; 14$.

11. Lee JY, Mucksavage $P$, Canales $C$, et al: High fidelity simulation based team training in urology: A preliminary interdisciplinary study of technical and nontechnical skills in laparoscopic complications management. J Urol 2012; 187: 1385-1391. Available at: http://dx.doi.org/10.1016/j.juro.2011.11.106.

12. Melnyk R, Ezzat B, Belfast E, et al: Mechanical and functional validation of a perfused, robot-assisted partial nephrectomy simulation platform using a combination of 3D printing and hydrogel casting. World J Urol 2019; 38: 1631-1641. Available at: https://doi.org/10.1007/s00345-019-02989-z.

13. Pradarelli JC, Gupta A, Hermosura AH, et al: Non-technical skill assessments across levels of US surgical training. Surg (United States) 2021. Available at: https://doi.org/10.1016/j.surg.2021.02.058.

14. Jung JJ, Yule S, Boet S, et al: Nontechnical Skill Assessment of the Collective Surgical Team Using the Non-Technical Skills for Surgeons (NOTSS) System. Ann Surg 2020; 272: 1158-1163. 
15. Eppich $\mathrm{W}$ and Cheng A: Promoting excellence and reflective learning in simulation (PEARLS): Development and rationale for a blended approach to health care simulation debriefing. Simul Healthc 2015; 10: 106-115.

16. Childs BS, Manganiello MD and Korets R: Novel Education and Simulation Tools in Urologic Training. Curr Urol Rep 2019; 20.

17. Rashid P and Gianduzzo TRJ: Urology technical and non-technical skills development: The emerging role of simulation. BJU Int 2016; 117: 9-16.

18. Young M, Kailavasan M, Taylor J, et al: The Success and Evolution of a Urological "Boot Camp" for Newly Appointed UK Urology Registrars: Incorporating Simulation, Nontechnical Skills and Assessment. J Surg Educ 2019; 76: 1425-1432. Available at: https://doi.org/10.1016/j.jsurg.2019.04.005.

19. Nguyen LN, Tardioli K, Roberts M, et al: Development and incorporation of hybrid simulation OSCE into intraining examinations to assess multiple canMEDS competencies in urologic trainees original research. J Can Urol Assoc 2015; 9: 32-36.

20. Hull L, Arora S, Aggarwal R, et al: The impact of nontechnical skills on technical performance in surgery: A systematic review. J Am Coll Surg 2012; 214: 214-230. Available at: http://dx.doi.org/10.1016/j.jamcollsurg.2011.10.016.

21. Yule S, Parker SH, Wilkinson J, et al: Coaching Non-technical Skills Improves Surgical Residents' Performance in a Simulated Operating Room. J Surg Educ 2015; 72: 11241130. Available at: http://dx.doi.org/10.1016/j.jsurg.2015.06.012.

22. Aggarwal R, Undre S, Moorthy K, et al: The simulated operating theatre: Comprehensive training for surgical teams. Qual Saf Heal Care 2004; 13: 27-32.

23. Doumouras AG, Keshet I, Nathens AB, et al: A crisis of faith? A review of simulation in teaching team-based, crisis management skills to surgical trainees. J Surg Educ 2012; 69: 274-281. Available at: http://dx.doi.org/10.1016/j.jsurg.2011.11.004.

24. Griffin C, Aydın A, Brunckhorst O, et al: Non-technical skills: a review of training and evaluation in urology. World J Urol 2019. Available at: https://doi.org/10.1007/s00345019-02920-6.

25. Van Cleynenbreugel BSEP, Gözen AS and Tokas T: The value of simulation-based training in the path to laparoscopic urological proficiency. Curr Opin Urol 2017; 27: 337-341.

26. Carrion DM, Rodriguez-Socarrás ME, Mantica G, et al: Current status of urology surgical training in Europe: an ESRU-ESU-ESUT collaborative study. World J Urol 2020; 38: 239-246. Available at: https://doi.org/10.1007/s00345-019-02763-1.

27. Brunckhorst O, Shahid S, Aydin A, et al: The relationship between technical and nontechnical skills within a simulation-based ureteroscopy training environment. J Surg Educ 2015; 72: 1039-1044. Available at: http://dx.doi.org/10.1016/j.jsurg.2015.04.002.

28. Chow AK, Sherer BA, Yura E, et al: Urology Residents' Experience and Attitude Toward Surgical Simulation: Presenting our 4-Year Experience With a Multiinstitutional, Multi-modality Simulation Model. Urology 2017; 109: 32-37. Available at: https://doi.org/10.1016/j.urology.2017.05.037. 
Figures and Tables

Fig. 1.

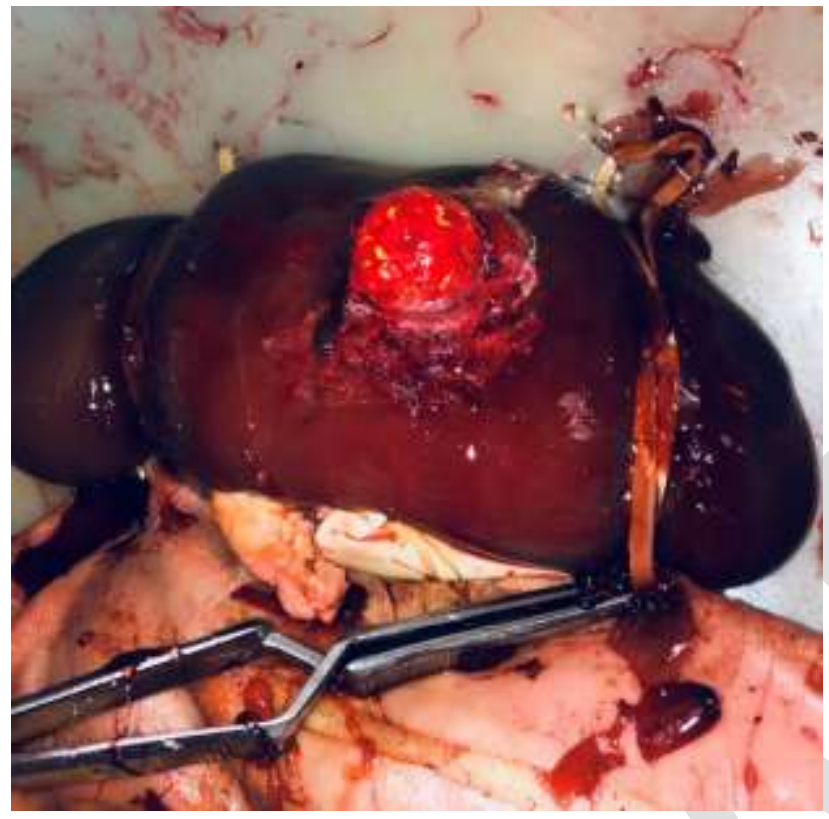

Fig. 2.

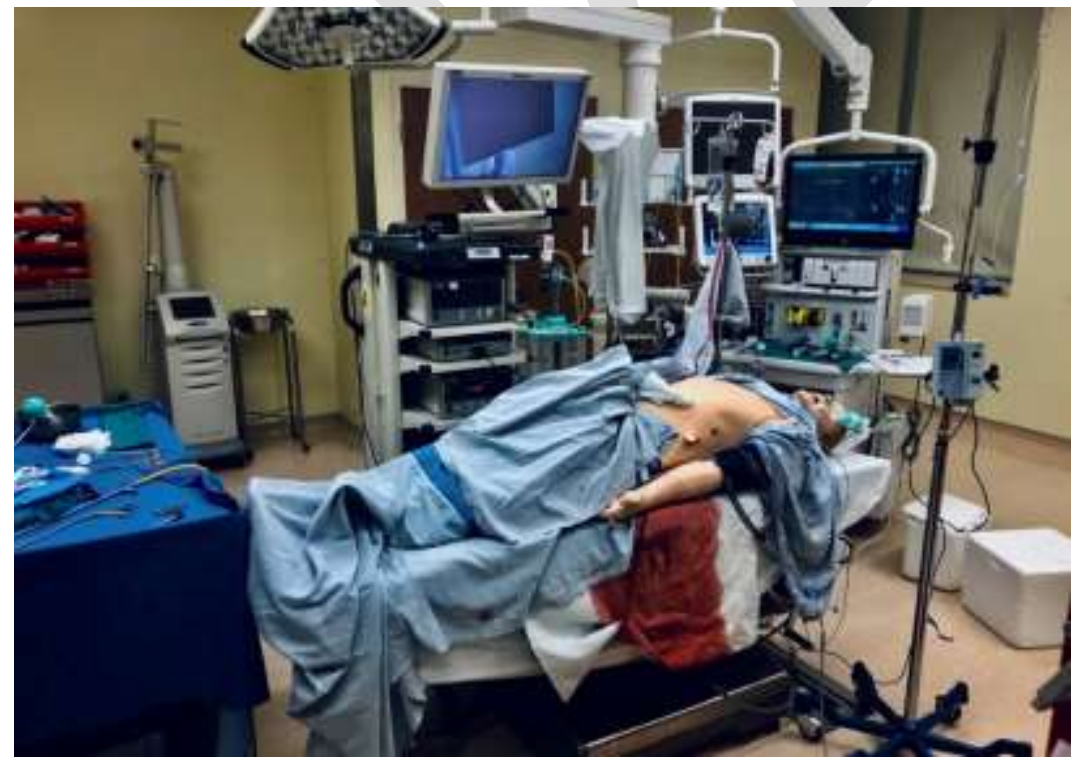




\begin{tabular}{|c|c|}
\hline Question & Response \\
\hline PGY level & $\begin{array}{l}\text { PGY } 3=2 \\
\text { PGY } 4=1 \\
\text { PGY } 5=4\end{array}$ \\
\hline $\begin{array}{l}\text { How many laparoscopic } \\
\text { simulations have you been } \\
\text { involved with (indicate a value } \\
\text { and not a range)? }\end{array}$ & $\begin{array}{l}\text { Resident } 1=2 \\
\text { Resident } 2=5 \\
\text { Resident } 3=0 \\
\text { Resident } 4=5 \\
\text { Resident } 5=0 \\
\text { Resident } 6=0 \\
\text { Resident } 7=1 \\
\text { Response range: } 0-5 \quad \text { Mean: } 1.9\end{array}$ \\
\hline $\begin{array}{l}\text { How many simulation-based } \\
\text { team training sessions have you } \\
\text { participated in (indicate a value } \\
\text { and not a range)? }\end{array}$ & $\begin{array}{l}\text { Resident } 1=0 \\
\text { Resident } 2=2 \\
\text { Resident } 3=5 \\
\text { Resident } 4=0 \\
\text { Resident } 5=5 \\
\text { Resident } 6=0 \\
\text { Resident } 7=5 \\
\text { Response range: } 0-5 \quad \text { Mean: } 2.4\end{array}$ \\
\hline $\begin{array}{l}\text { Goal of the simulation-based } \\
\text { team training sessions? }\end{array}$ & $\begin{array}{l}\text { 1. Communication skills } \\
\text { 2. Lap skills and interdisciplinary communication } \\
\text { 3. Problem solving in unwell patient and interdisciplinary } \\
\text { communication } \\
\text { 4. ATLS-intro to surgery and POS } \\
\text { 5. Communication skills, critical care, ACLS, trauma }\end{array}$ \\
\hline $\begin{array}{l}\text { Have you ever encountered a } \\
\text { bleeding laparoscopic partial or } \\
\text { radical nephrectomy in real life } \\
\text { that needed to be converted to an } \\
\text { open procedure; if so, how } \\
\text { many? }\end{array}$ & $\begin{array}{l}\mathrm{Y}: 4 \\
\text { a. Number encountered: Each encountered a single event } \\
\mathrm{N}: 3\end{array}$ \\
\hline $\begin{array}{l}\text { What was most useful to you } \\
\text { today? }\end{array}$ & $\begin{array}{l}\text { 1. Ways to improve communication with other professionals } \\
\text { 2. Working with anaesthesia } \\
\text { 3. Identifying communication barriers during stressful OR cases }\end{array}$ \\
\hline
\end{tabular}




\begin{tabular}{|c|c|}
\hline & $\begin{array}{l}\text { 4. Practicing difficult scenario and direct this to other scenarios } \\
\text { (e.g., prone) } \\
\text { 5. Practice laparoscopic troubleshooting in an acute bleeding } \\
\text { scenario } \\
\text { 6. Communication aspect with anaesthesia } \\
\text { 7. Very realistic simulation which made my responses real. } \\
\text { Reminded me of the importance of anticipating critical } \\
\text { situations }\end{array}$ \\
\hline $\begin{array}{l}\text { Will you be able to implement } \\
\text { what you learned today in your } \\
\text { practice? How? }\end{array}$ & $\begin{array}{l}\text { 1. Yes-communication tips } \\
\text { 2. Yes-common procedure in urology. This is a definite } \\
\text { complication/event. Great practice! } \\
\text { 3. Yes - will be more aware of communicating issues to } \\
\text { anaesthesia colleagues } \\
\text { 4. Yes - practice clear communication. } \\
\text { 5. Yes - more communication skills with anaesthesia; more } \\
\text { communication is better; opening is an option for hemorrhage } \\
\text { during lap case } \\
\text { 6. Yes - being on the same page as anaesthesia during crash } \\
\text { scenarios } \\
\text { 7. Yes-increase my communication skills with the anaesthesia } \\
\text { team }\end{array}$ \\
\hline $\begin{array}{l}\text { Where do you train your } \\
\text { leadership and teamwork skills } \\
\text { in residency? }\end{array}$ & $\begin{array}{l}\text { 1. Daily activities } \\
\text { 2. In OR and mentoring junior residents in clinical settings } \\
\text { 3. In OR, when on-call, during rounds } \\
\text { 4. On the job-OR, ward, ER } \\
\text { 5. OR and wards } \\
\text { 6. While we're working (no formal training) } \\
\text { 7. Running the inpatient service and watching juniors }\end{array}$ \\
\hline
\end{tabular}

